\title{
Differential responses of mast cell Toll-like receptors 2 and 4 in allergy and innate immunity
}

\author{
Volaluck Supajatura, ${ }^{1,2}$ Hiroko Ushio, ${ }^{2}$ Atsuhito Nakao, ${ }^{2}$ Shizuo Akira, ${ }^{3}$ Ko Okumura, ${ }^{2,4}$ \\ Chisei Ra, ${ }^{5}$ and Hideoki Ogawa ${ }^{1,2}$
}

${ }^{1}$ Department of Dermatology, and

${ }^{2}$ Atopy (Allergy) Research Center, Juntendo University School of Medicine, Tokyo, Japan

${ }^{3}$ Department of Host Defense, Research Institute for Microbial Diseases, Osaka University, Osaka, Japan

${ }^{4}$ Department of Immunology, Juntendo University School of Medicine, Tokyo, Japan

${ }^{5}$ Advanced Medical Research Center, Nihon University School of Medicine, Tokyo, Japan

Address correspondence to: Hiroko Ushio, Atopy (Allergy) Research Center,

Juntendo University School of Medicine, 2-1-1 Hongo, Bunkyo-ku, Tokyo 113-8421, Japan.

Phone: 81-3-5802-1591; Fax: 81-3-3813-5512; E-mail: hushio@med.juntendo.ac.jp.

Received for publication November 26, 2001, and accepted in revised form April 10, 2002.

Toll-like receptor 2 (TLR2) and TLR4 play important roles in the early innate immune response to microbial challenge. To clarify the functional roles of TLRs 2 and 4 in mast cells, we examined bone marrow-derived mast cells (BMMCs) from TLR2 or TLR4 gene-targeted mice. Peptidoglycan (PGN) from Staphylococcus aureus stimulated mast cells in a TLR2-dependent manner to produce TNF- $\alpha$, IL-4, IL-5, IL-6, and IL-13, but not IL-1 $\beta$. In contrast, LPS from Escherichia coli stimulated mast cells in a TLR4-dependent manner to produce TNF- $\alpha$, IL-1 $\beta$, IL-6, and IL-13, but not IL-4 nor IL-5. Furthermore, TLR2- but not TLR4-dependent mast cell stimulation resulted in mast cell degranulation and $\mathrm{Ca}^{2+}$ mobilization. In a mast cell-dependent model of acute sepsis, TLR4 deficiency of BMMCs in mice resulted in significantly higher mortality because of defective neutrophil recruitment and production of proinflammatory cytokines in the peritoneal cavity. Intradermal injection of PGN led to increased vasodilatation and inflammation through TLR2-dependent activation of mast cells in the skin. Taken together, these results suggest that direct activation of mast cells via TLR2 or TLR4 by respective microligands contributes to innate and allergic immune responses.

J. Clin. Invest. 109:1351-1359 (2002). doi:10.1172/JCI200214704.

\section{Introduction}

Mast cells are particularly frequent in close proximity to epithelial surfaces where they are strategically located for optimal interaction with the environment and for their putative functions in host defense $(1,2)$. Mast cells have been viewed primarily as effector cells of anaphylactic reactions but also viewed as important cells for initiation of innate and acquired immune response against various pathogens (3-5). These reactions are mediated by a number of preformed or newly synthesized mediators and cytokines that are secreted upon activation of mast cells (6). In contrast to the well-known activation of mast cells via high-affinity IgE receptors (FcERI), the mechanisms of direct activation of mast cells by various microorganisms have not been studied precisely.

Recent reports suggest that innate immune responses are also initiated by certain pattern recognition receptors, called Toll-like receptors (TLRs). TLRs comprise a family of mammalian cell-surface proteins that stimulate proinflammatory cytokine gene transcription in response to various microbial ligands (7). A large body of evidence studied in TLR2 and TLR4 gene-targeted mice indicated that TLR4 is a signal transducer of LPS, a component of Gram-negative bacteria (GNB) (8-10), whereas TLR2 appears to mediate responses to lipoteichoic acid, lipopeptides, and peptidoglycan (PGN) from
Gram-positive bacteria (GPB) and mycobacteria (11-17). We have previously demonstrated, using LPS-low responder $\mathrm{C} 3 \mathrm{H} / \mathrm{HeJ}$ mice, that there are expressions of TLRs on mast cells and that mast cell TLR4 mediates innate immune response against GNB (18). However, to date, it has still been undefined whether the recognition of pathogens by each TLR on the mast cell is similar to that on the other leukocytes, what roles are played by TLRs in the function of mast cells, and if there are quantitative or qualitative differences in the mast cell activation by different TLRs. Staphylococcus aureus, the bestknown GPB, colonizes on more than $90 \%$ of atopic eczematous skin, and the number of colonized bacteria and an increased number of activated mast cells are well correlated with the severity of the eczematous lesion (19, 20). Since mast cells express TLR2, it is important to clarify whether the clinical features are dependent on the actual activation of mast cells via TLR 2 by GPB.

In this study, we first show that mast cell TLR2 or mast cell TLR4 recognizes the pathogen ligands in a way similar to those of other leukocytes; that is, mast cell TLR2 but not mast cell TLR 4 confers GPB component responsiveness, and TLR4 but not TLR2 is sufficient for GNB responsiveness. Second, we observe quantitative and qualitative differences in the activation of mast cells via TLR2 and TLR4 stimulation. TLR2-mediated mast cell 
activation by PGN leads to degranulation and IL- 4 and IL-5 cytokine production, in contrast to TLR4-mediated mast cell activation, in which TNF- $\alpha$, IL-1 $\beta$, IL- 6 , and IL-13 are the major cytokines produced. Finally, we provide new insights into the different roles played by mast cells in vivo in a certain environment. TLR4-mediated activation of peritoneal mast cells is crucial for host protection from Gram-negative bacterial infection, whereas TLR2-mediated activation of skin mast cells causes acute and late reactions by PGN application and may exacerbate the inflammatory lesions of atopic dermatitis, in which $S$. aureus infection is common.

\section{Methods}

Mice. TLR2-deficient (TLR2-/-), TLR4-deficient (TLR4-/-), and corresponding wild-type (TLR2 ${ }^{+/+}$or TLR $4^{+/+}$) mice were kindly provided by S. Akira (Osaka University) (9, 12). WBB6 $F_{1}-W / W^{v}$ mice were purchased from Japan SLC (Hamamatsu, Japan). All animal experiments were performed according to the approved manual of the Institutional Review Board of Juntendo University.

Generation of bone marrow-derived mast cells. Bone marrow-derived mast cells (BMMCs) were generated from the femoral bone marrow cells of mice and maintained in the presence of $10 \%$ pokeweed mitogen-stimulated spleen-conditioned medium as a source of mast cell growth factors as previously described $(18,21)$. After 4 weeks of culture, more than $99 \%$ of the cells were identifiable as mast cells as determined by Toluidine blue staining and FACS analysis of cell surface expression of $c-k i t$ and FceRI.

$\beta$-Hexosaminidase release assay. A total of $5 \times 10^{5}$ BMMCs $/ \mathrm{ml}$ in Tyrode's buffer ( $10 \mathrm{mM}$ HEPES buffer [pH 7.4], $130 \mathrm{mM} \mathrm{NaCl}, 5 \mathrm{mM} \mathrm{KCl}$, and $5.6 \mathrm{mM}$ glucose) containing $10 \%$ FCS (as a source of soluble CD14), $1 \mathrm{mM} \mathrm{CaCl}_{2}$, and $0.6 \mathrm{mM} \mathrm{MgCl}_{2}$ were stimulated with the indicated concentrations of PGN from S. aureus (Sigma-Aldrich, Tokyo, Japan) or LPS from Escherichia coli (serotype 0111:B4; Sigma-Aldrich) for 1 hour at $37^{\circ} \mathrm{C}$. The $\beta$-hexosaminidase in the supernatants and cell lysate was quantified by hydrolysis of $p$-nitrophenyl- $N$-acetyl- $\beta$-D-glucopyranoside (SigmaAldrich) in $0.1 \mathrm{M}$ sodium citrate buffer ( $\mathrm{pH} 4.5)$ for 90 minutes at $37^{\circ} \mathrm{C}$. The percentage of $\beta$-hexosaminidase release was calculated as previously described (18).

Intracellular $\mathrm{Ca}^{2+}$ mobilization. BMMCs $\left(1 \times 10^{6}\right.$ cells $/ 200 \mu \mathrm{l}$ ) were incubated under condition of darkness with $5 \mu \mathrm{M}$ of fura- $2 \mathrm{AM}$ (Dojindo Laboratories, Kumamoto, Japan) for 30 minutes, at $37^{\circ} \mathrm{C}$ in complete culture medium. Cells were washed with excess amounts of cold Tyrode's buffer and then resuspended in Tyrode's buffer containing $1 \mathrm{mM} \mathrm{CaCl}_{2}$ and 0.6 $\mathrm{mM} \mathrm{MgCl}_{2}$ at the concentration of $5 \times 10^{5}$ cells $/ \mathrm{ml}$. Cells were stimulated with $100 \mu \mathrm{g} / \mathrm{ml}$ of PGN at $37^{\circ} \mathrm{C}$. BMMCs stimulated with IgE and anti-IgE were used as positive control. Intracellular $\mathrm{Ca}^{2+}$ mobilization was monitored at a $510 \mathrm{~nm}$ emission wavelength excited by 340 and $360 \mathrm{~nm}$ using a fluorescence spectrophotometer model F2000 (Hitachi Ltd., Tokyo, Japan).
Measurement of cytokine concentrations. BMMCs $\left(1 \times 10^{6}\right.$ cells $/ \mathrm{ml}$ ) in complete culture medium were stimulated with the indicated concentration of PGN or LPS. Cells were incubated at $37^{\circ} \mathrm{C}, 3$ hours for TNF- $\alpha$ or 6 hours for IL-1 $\beta$, IL-4, IL-5, IL-6, and IL-13 (a preliminary experiment showed that these time points were optimal for the production of each cytokine from mast cells). The level of each cytokine in supernatant was measured by ELISA kit (Genzyme Techne Corp., Minneapolis, Minnesota, USA).

Western blot analysis. A total of $5 \times 10^{6} \mathrm{BMMCs} / \mathrm{ml}$ were stimulated with PGN $(100 \mu \mathrm{g} / \mathrm{ml})$ or LPS $(50 \mathrm{ng} / \mathrm{ml})$ for the indicated time period. The reaction was stopped with cold Tyrode's $\left(\mathrm{CA}^{2+}, \mathrm{MG}^{2+}\right.$ free) buffer. The cells were lysed with $20 \mu$ l of lysis buffer ( $1 \%$ Triton X-100, $150 \mathrm{mM}$ $\mathrm{NaCl}, 25 \mathrm{mM}$ Tris- $\mathrm{HCl}$ [pH 7.5], $1 \mathrm{mM}$ EDTA, containing $1 \mu \mathrm{M}$ PMSF, $10 \mu \mathrm{g} / \mathrm{ml}$ leupeptin, $10 \mu \mathrm{g} / \mathrm{ml}$ pepstatin- $\mathrm{A}, 50 \mu \mathrm{g} / \mathrm{ml}$ aprotinin, and $2 \mathrm{mM}$ sodium orthovanadate), and the lysates were subjected to $10 \%$ SDS-PAGE (Bis Tris; Novex, San Diego, California, USA). The immunoblotting using polyclonal antibody to tyrosine-phosphorylated IкB- $\alpha$ (New England Biolabs Inc., Beverly, Massachusetts, USA) and IкB- $\alpha$ (New England Biolabs Inc.) was done according to the manufacturer's instructions (18). The membrane was developed with an enhanced chemiluminescence detection kit (Amersham Pharmacia Biotech, Piscataway, New Jersey, USA).

Reconstitution of $W / W^{v}$ mice with BMMCs. Mast cell deficiency of $W / W^{v}$ mice in the peritoneal cavity was selectively reconstituted by the injection, 4 weeks after starting the culture, of $2 \times 10^{6} \mathrm{BMMCs}$ from TLR4-/, TLR2 ${ }^{-/}$, or respective wild-type mice into the peritoneal cavity as previously described $(18,22)$. For the reconstitution of skin mast cells of $W / W^{v}$ mice, $1 \times 10^{6}$ BMMCs from TLR4 ${ }^{-/}$, TLR2 ${ }^{-/}$, or respective wild-type mice, in $20 \mu \mathrm{l}$ of normal saline, were injected intradermally into the ears $(22,23) 4$ weeks after starting the culture. Five weeks after injection of BMMCs, the mice were used for experiments. Reconstitution of mast cells was confirmed by Toluidine blue or Alcian blue and Safranin staining of the cytospun preparation of peritoneal cells or formalin-fixed tissue section of the skin.

Cecal ligation and puncture. Cecal ligation and puncture (CLP) was performed as previously described (18). Briefly, mice were anesthetized with sodium pentobarbital and the cecum was isolated, ligated with 4-0 silk, and punctured once with a 21-gauge needle. After CLP, mice were observed for mortality at least five times daily over a period of 10 days. Before CLP was performed, the mice were coded so that the CLP was done without notifying individual groups.

Differential cell counts and estimation of cytokine concentrations in peritoneal exudates. Peritoneal exudates were collected from CLP-induced mice at the indicated time points, and total cell numbers were counted. Cytospun preparations were made from the exudates of each mouse, and differential cell counts of infiltrating leukocytes were done by counting 500 leukocytes under oil immersion fields after staining with Diff-Quik (International Reagents, Kobe, Japan). The percentage of 
mast cells in the exudates was determined by Toluidine blue ( $\mathrm{pH} 4.0)$ or Alcian blue and Safranin staining of cytospun preparation. The levels of cytokines in peritoneal fluids were determined by ELISA kits according to the manufacturer's instruction (Genzyme Techne).

Induction of skin inflammation. Mice were lightly anesthetized with ether, and $20 \mu \mathrm{l}$ of PGN $(100 \mu \mathrm{g} / \mathrm{ml}$ in saline) or LPS (100 $\mathrm{ng} / \mathrm{ml}$ in saline) was injected intradermally into the left ear. The vehicle saline was injected into the right ear as a control. To visualize vascular leakage, $250 \mu \mathrm{l}$ of $0.5 \%$ Evans blue in saline was injected intravenously, 5 minutes before PGN or LPS application. After 15 minutes of application, mice were sacrificed and ears were removed. The amount of dye in the ear was measured as previously described with slight modification (24). An ear was dissolved in $200 \mu \mathrm{l}$ of $1 \mathrm{~N}$ $\mathrm{KOH}$ solution overnight at $37^{\circ} \mathrm{C}$, and $1.8 \mathrm{ml}$ of a mixture of $0.6 \mathrm{~N} \mathrm{H}_{3} \mathrm{PO}_{4}$ solution and acetone (5:13) was added. After vigorous shaking, the supernatant was collected by centrifugation at $1920 \mathrm{~g}$ for 10 minutes and the optical density was measured at $620 \mathrm{~nm}$. The amount of dye was calculated according to the standard curve of known concentration of Evans blue. For histological examination, ears were removed 15 minutes or 4 hours after PGN or LPS application and fixed in buffered formalin and then stained with hematoxylin and eosin $(\mathrm{H} \& \mathrm{E})$ or Toluidine blue. The number of dermal mast cells and neutrophils present at the reaction sites was determined as previously described $(25,26)$ and expressed as the number of cells per square millimeter of dermis. The mast cells were classified into three categories: extensively degranulated $(50 \%$ of the cytoplasmic granules exhibiting fusion, staining alterations, and extrusion from the cell), slightly to moderately degranulated $(10-15 \%$ of the granules exhibiting fusion or discharge), or normal $(25,26)$.

Statistical analysis. Statistical analysis was performed using the Student $t$ test. Statistical analysis of survival data in the CLP experiment was performed using the Logrank test.

\section{Results}

PGN but not LPS induces mast cell degranulation and $\mathrm{Ca}^{2+}$ mobilization via TLR2. To investigate the involvement of TLR2 and TLR4 in bacterial microorganism-induced mast cell activation, first we investigated the direct effects of these microbial ligands on mast cell degranulation, a major function of mast cells. When evaluated by $\beta$-hexosaminidase release, PGN induced degranulation of BMMCs from TLR2-bearing mice, which was almost comparable to that of IgE receptor crosslinking (Figure 1a). In contrast, the same concentration of PGN did not induce any release of $\beta$-hexosaminidase from TLR2 $-/-$ BMMCs, even though these BMMCs released a similar amount of $\beta$-hexosaminidase with TLR2-bearing BMMCs upon IgE receptor cross-linking and calcium ionophore stimulation. This reaction was mediated by TLR 2 , since TLR4 $4^{-/}$and TLR $4^{+/+}$mice showed responses similar to that of TLR2 $2^{+/}$mice (Fig- ure 1a). Consistent with previous data, LPS stimulation did not cause degranulation of mast cells from any strains of mice $\left(\mathrm{TLR}^{+/+}\right.$, TLR2 ${ }^{-/-}$, TLR4 ${ }^{+/+}$, TLR4 ${ }^{-/}$, $\mathrm{C} 57 \mathrm{BL} / 6, \mathrm{BALB} / \mathrm{c}, \mathrm{C} 3 \mathrm{H} / \mathrm{HeN}, \mathrm{C} 3 \mathrm{H} / \mathrm{HeJ}$, and NC/Nga; Figure 1a and data not shown). Degranulation of BMMCs was well correlated with intracellular $\mathrm{Ca}^{2+}$ mobilization. Upon PGN stimulation, intracellular increase of $\mathrm{Ca}^{2+}$ was observed in TLR2 $2^{+/+}$but not in TLR2-/- BMMCs. The concentration of intracellular $\mathrm{Ca}^{2+}$ reached a maximum level within 50 seconds after PGN stimulation, and no significant difference was observed in the pattern of $\mathrm{Ca}^{2+}$ mobilization compared with FcERI cross-linking (Figure 1b). The effect of PGN on mast cells was not because of the cytotoxic effect, since these concentrations of PGN did not show any cytotoxicity to BMMCs as evaluated by lactate dehydrogenase release assay.

Different pattern of cytokine production by mast cells upon activation via TLR 2 or TLR4. Next we investigated whether PGN or LPS could activate mast cells to secrete cytokines via respective TLRs, since it has been reported that activation of mast cells leads to the secretion of preformed and newly synthesized cytokines (27). The BMMCs from TLR2 ${ }^{+/+}$, TLR $4^{+/+}$, and TLR4 $4^{-/-}$mice did produce similar levels of IL-4, IL-5, IL-6, IL-13, and TNF- $\alpha$ in response to PGN in a dose-dependent manner (Figure 2a). In contrast, BMMCs from TLR2 ${ }^{-/-}$mice did not produce any of these cytokines upon PGN stimulation. Since BMMCs from TLR $4^{+/+}$or TLR $4^{-/-}$mice could produce similar levels of cytokines, the absence of TLR4 did not influence the responses of BMMCs to PGN. Also, the TLR4-bearing BMMCs could produce TNF- $\alpha$, IL- $1 \beta$, IL- 6 , and IL-13 in response to LPS in a dose-dependent manner (Figure $2 b$ ). These responses were completely deficient in TLR4 $/$ - BMMCs. The pattern and levels of these cytokines released by $\mathrm{TLR} 2^{+/+}$or TLR2 ${ }^{-/}$BMMCs were not significantly different from those of TLR4 ${ }^{+/+}$BMMCs, suggesting that the absence of TLR2 does not influence the function of TLR4 and that the TLR4 molecule is, at least, a principal LPS signal transducer in mast cells. These results were consistent with our previous work, which used BMMCs from $\mathrm{C} 3 \mathrm{H} / \mathrm{HeJ}$ mice and showed that intact TLR 4 is required for full activation of mast cells by LPS (18).

Stimulation of mast cells via both TLR2 and TLR4 leads to activation of NF- $\kappa B$ signaling. The cytoplasmic domain of members of the TLR family is homologous to that of the IL-1 receptor (IL-1R), which subsequently drives the transcriptional induction of several cytokine genes after activation of NF- $\mathrm{KB}(28)$. We determined whether NF- $\kappa B$ activation was also triggered in mast cells upon PGN or LPS stimulation. The phosphorylation of

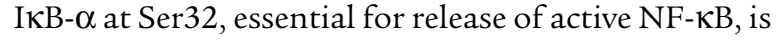
a marker of NF- $\kappa B$ activation. PGN $(100 \mu \mathrm{g} / \mathrm{ml})$ and LPS $(50 \mathrm{ng} / \mathrm{ml})$ induced the phosphorylated IкB- $\alpha$ in a TLR2- or TLR4-dependent manner, respectively. The activity of NF- $\mathrm{\kappa B}$ in mast cells was at maximum 15 minutes after both stimulations and then gradually decreased within 30 minutes (Figure 3). These results 

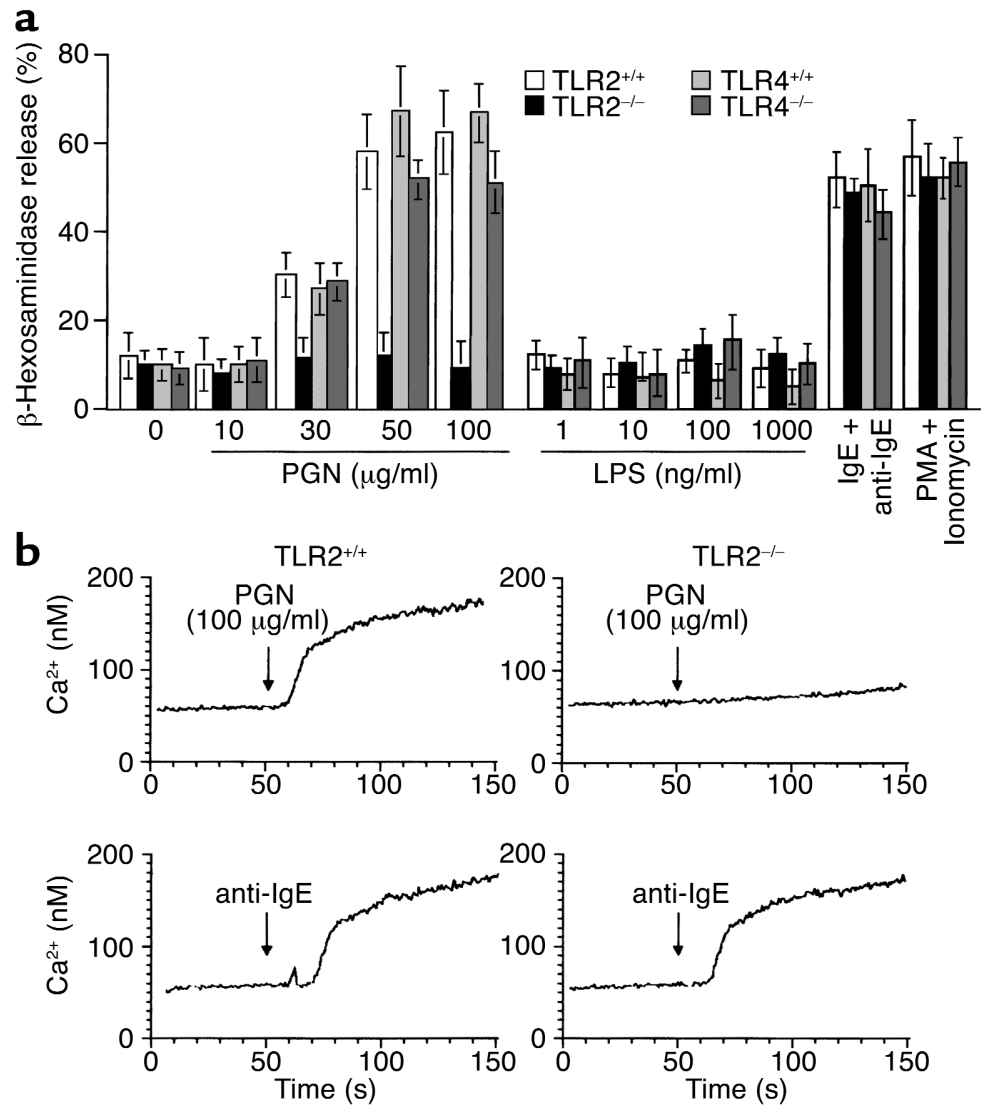

Figure 1

PGN but not LPS induces mast cell degranulation and $\mathrm{Ca}^{2+}$ mobilization through TLR2. (a) BMMCs from $\mathrm{TLR}^{+/+}$(white bars), TLR2-/- (black bars), TLR4 $4^{+/+}$ (light gray bars), and TLR4 ${ }^{--}$(dark gray bars) were incubated with indicated concentrations of PGN or LPS. $\beta$-Hexosaminidase in the supernatant was measured as described in Methods. Data shown are mean \pm SD of three experiments conducted with different BMMC preparations. BMMCs stimulated with IgE and anti-IgE or PMA and ionomycin were used as positive control. (b) $\mathrm{TLR}^{+/+}$and $\mathrm{TLR}^{-/-}$BMMCs were stimulated with $100 \mu \mathrm{g} / \mathrm{ml}$ of PGN; then $\mathrm{Ca}^{2+}$ mobilization was evaluated as described in Methods. BMMCs stimulated with IgE and anti-IgE were used as positive control. Data shown is one representative result from several experiments that had similar results. suggest that both PGN-TLR2 and LPS-TLR4 signaling in mast cells is followed by activation of NF- $\mathrm{KB}$.

Mast cell TLR4 is required for protection of mice from CLPinduced acute bacterial infection. Using the mast cell-dependent acute septic peritonitis model, we clarified the importance of mast cell TLR4 for bacterial infection in vivo. Peritoneal mast cells of mast cell-deficient $W / W^{v}$ mice were reconstituted with BMMCs from $\mathrm{TLR}^{+/+}$, TLR4 ${ }^{-/-}$, TLR2 ${ }^{+/+}$, or TLR2 $2^{-/-}$mice and subjected to CLP. Some mast cell-deficient $W / W^{v}$ mice died on the first day after CLP; $100 \%$ died within 3 days (Figure 4a). Some of the $W / W^{v}$ mice reconstituted with TLR4deficient BMMCs (W/W $\mathrm{W}^{v} / \mathrm{TLR}^{-/-}$) died within 5 days; only 30\% survived 5 days after CLP. In contrast, $80-100 \%$ of $W / W^{v}$ mice reconstituted with $\mathrm{TLR}^{+/+}$BMMCs $\left(W / W^{v} / \mathrm{TLR}^{+/+}\right)$, TLR2 ${ }^{+/+}$BMMCs $\left(W / W^{v} / \mathrm{TLR}^{+/+}\right)$, or TLR2 $2^{--}$BMMCs (W/W $W^{v} /$ TLR2 $\left.^{-/-}\right)$survived during the experimental periods. Three days after CLP, $W / W^{v} /$ TLR $^{+/+}, W / W^{v} /$ TLR2 $^{+/+}$, and $W / W^{v} /$ TLR2 $^{-/-}$mice showed a significantly higher survival rate than $W / W^{v}$ and $W / W^{v} /$ TLR $4^{-/-}$mice $(P<0.05)$. This result is similar to the previous result of experiments using $\mathrm{C} 3 \mathrm{H} / \mathrm{HeJ}$-derived BMMCs for reconstitution of $\mathrm{W} / \mathrm{W}^{v}$ mice (18), and there were no significant differences in the survival rate between $W / W^{v} /$ TLR2 $^{+/+}$and $W / W^{v} /$ TLR2 $^{-/-}$, indicating again that mast cells play an important role in host protection from early enterobacterial infection via the TLR4 molecule but not the TLR2. Five weeks after reconstitution, $W / W^{v} / \mathrm{TLR}^{+/+}, W / W^{v} / \mathrm{TLR}^{-/-}$,
$W / W^{v} /$ TLR2 $^{+/+}$, and $W / W^{v} /$ TLR2 $2^{-/-}$mice had no significant differences in the number of peritoneal mast cells $\left(4.56 \times 10^{4} \pm 0.50 \times 10^{4}, 4.77 \times 10^{4} \pm 0.37 \times 10^{4}\right.$, $4.61 \times 10^{4} \pm 0.37 \times 10^{4}$, and $3.57 \times 10^{4} \pm 0.95 \times 10^{4}$ cells per mouse, respectively). More than $95 \%$ of these mast cells showed positive staining with Alcian blue and Safranin, which was the property of connective tissue mast cells. Their function evaluated by $\beta$-hexosaminidase release after FceRI cross-linking was the same (data not shown).

Deficiency of mast cell TLR4 results in defective neutrophil recruitment and proinflammatory cytokine production in the peritoneal cavity after CLP. Since it has been reported that early leukocyte, especially neutrophil, recruitment into the peritoneal cavity after CLP is crucial for protection of mice from acute septic peritonitis-induced death, and that this step is highly dependent on mast cell activation by enterobacteria, we examined whether the defective leukocyte recruitment in the peritoneal cavity after CLP was associated with the deficiency of mast cell TLR4. Although there was no significant difference in the number of leukocytes in peritoneal fluid in $W / W^{v}$, W/Wv $/$ TLR $^{+/+}$, W/Wv $/$TLR $^{-/-}$, W/W $W^{v} /$ TLR2 $^{+/+}$, and $W / W^{v} /$ TLR2 ${ }^{-/-}$mice at 5 weeks after reconstitution, the number of leukocytes (most of them were neutrophils) in peritoneal exudates was significantly higher in $W / W^{v} /$ TLR $^{+/+}, W / W^{v} /$ TLR2 $^{+/+}$, and $W / W^{v} /$ TLR2 $^{-/-}$mice than in $W / W^{v} /$ TLR $4^{-/-}$and $W / W^{v}$ mice 6 hours after CLP $(P<0.01)$ (Figure 4b). These results were correlated with 
the proinflammatory cytokine production in the peritoneal cavity. The levels of TNF- $\alpha$, IL-1 $1 \beta$, IL- 6 , and IL-13 in peritoneal cavities of $W / W^{v} / \mathrm{TLR}^{+/+}, W / W^{v} / \mathrm{TLR}^{+/+}$, and $W / W^{w} /$ TLR2 $^{-/-}$mice 6 hours after CLP were significantly higher than those of $W / W^{v}$ and $W / W^{v} /$ TLR $^{-/-}$ mice $(P<0.01$ in each cytokine) (Figure $4 c)$.

TLR2 on mast cell-dependent acute skin responses to PGN. To study whether mast cell TLR2 is a physiologically relevant receptor for PGN in vivo, we compared the ability of intradermal injection of PGN to cause acute and late skin reactions in $W / W^{v}$ mice and $W / W^{v}$ mice reconstituted with TLR2 $/-$ or TLR4 ${ }^{-/}$BMMCs. The vascular permeability, evaluated by extravasation of Evans blue dye, was increased significantly by PGN $(P<0.001)$ but not LPS application in wild-type mice compared with vehicle control (Figure 5a). This reaction was dependent on the skin mast cells, since mast cell-deficient $W / W^{v}$ mice showed significantly less reaction $(P<0.001)$ (Figure $5 \mathrm{~b}$ ) and reconstitution of skin mast cells with BMMCs from wild-type mice increased the extravasation of the dye almost to the level of normal mice. This reaction was also mediated by TLR2, since TLR2 $2^{-/-}$mice and $W / W^{v}$ mice whose skin mast cells were reconstituted with intradermal injections of TLR2 ${ }^{-/}$BMMCs did not show significant response to PGN $(P<0.001)$. The reactions of TLR4 ${ }^{-/-}$mice and TLR4 ${ }^{-/}$BMMC-reconstituted mice were not significantly different from those of wild-type mice and mice reconstituted with wild-type BMMCs (Figure 5b).

The mast cells in the skin 5 weeks after reconstitution showed no differences in number between $W / W^{v} /$ wildtype $\left(W / W^{v} / W T\right), W / W^{v} /$ TLR4 $-/$, and $W / W^{v} /$ TLR2 $^{-/}$ mice $(15.8 \pm 4.4,15.2 \pm 4.9$, and $17.8 \pm 8.0$ per linear millimeter of ear skin, respectively), and they were both Alcian blue- and Safranin-positive, suggesting that they were connective tissue-type mast cells (Figure 5c). The histopathologic changes of the skin were investigated at 15 minutes and 4 hours after PGN or LPS injection. PGN challenge to $W / W^{v} / W T$ and $W / W^{v} /$ TLR4-/- but not $W / W^{v} /$ TLR2 ${ }^{-1-}$ mice resulted in degranulation of skin mast cells (Figure $5 \mathrm{~d}$, left column). The local vasodilatation began at 5 minutes after PGN injection in $W / W^{w} / W T$ and $W / W^{v} /$ TLR4 $4^{-/}$but not $W / W^{v} /$ TLR2 ${ }^{-/-}$or $W / W^{v}$ mice, and vasodilatation gradually increased,
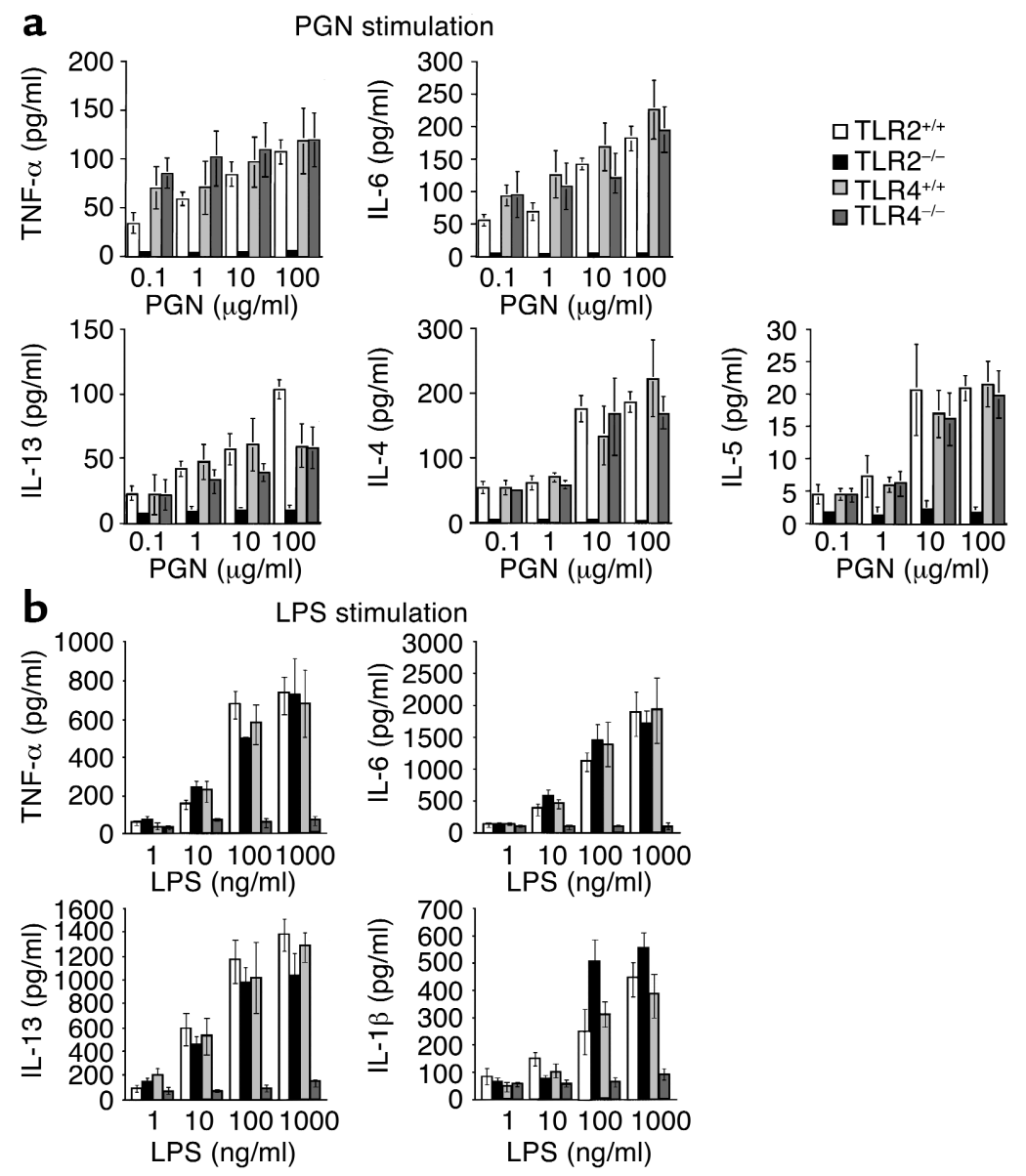

Figure 2

PGN-TLR2 stimulation and LPS-TLR4 stimulation lead to different cytokine production by BMMCs. BMMCs from TLR2 ${ }^{+/+}$(white bars), $\mathrm{TLR}^{-{ }^{--}}$(black bars), TLR4 ${ }^{+/+}$(light gray bars), and TLR4 ${ }^{-/}$(dark gray bars) were stimulated with indicated concentrations of PGN (a) or LPS (b), 3 hours for TNF- $\alpha$ and 6 hours for other cytokines. The concentrations of cytokine in the supernatant were determined as described in Methods. Data shown are mean \pm SD of three experiments conducted with different BMMC preparations. 


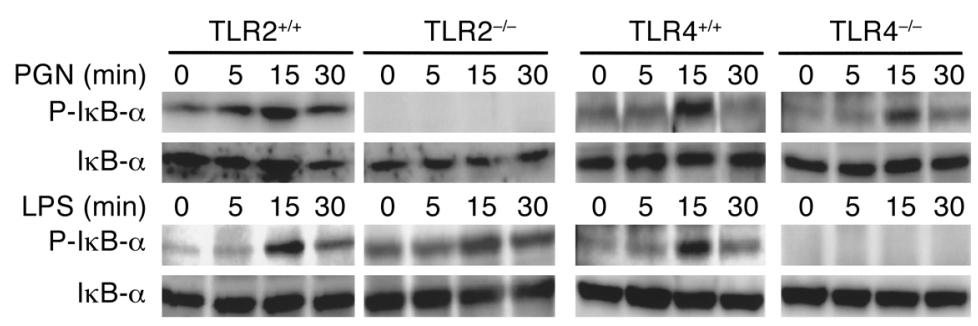

Figure 3

Stimulation of mast cells via both TLR2 and TLR4 leads to activation of NF-KB signaling. BMMCs were stimulated with $100 \mu \mathrm{g} / \mathrm{ml}$ of PGN or $50 \mathrm{ng} / \mathrm{ml}$ of LPS for 5,15 , and 30 minutes. Then lysates were subjected to SDS-PAGE and immunoblotted with antibody to phosphorylated IKB- $\alpha(P-\mid \kappa B-\alpha)$. The same blots were stripped and reblotted with antibody to IKB- $\alpha$. The result shown is a representative of three experiments conducted with different BMMC preparations that had similar results.

becoming marked at 15 minutes, accompanied by granulocyte infiltration in which the predominant cells were neutrophils (Figure 5d, middle column). Four hours after injection, wheal reaction was decreased but persistent accumulation of neutrophils was still obvious (Figure $5 \mathrm{~d}$, right column). In contrast, saline-injected skin had intact mast cells without degranulation in all mice (Figure 5c, bottom row). These reactions, especially degranulation and vasodilatation, were specific to PGN challenge, since LPS did not cause degranulation of mast cells nor vasodilatation of the skin at $15 \mathrm{~min}$ utes (Figure 5d, bottom row). Interestingly, LPS caused some accumulation of neutrophils 4 hours after application, but the number of accumulating neutrophils was much less than after PGN application (Figure $5 \mathrm{~d}$ and Table 1). These histopathological changes (infiltration of neutrophils and mast cell degranulation) were quantitatively evaluated and are shown in Table 1.

\section{Discussion}

This study clearly shows that mast cells can function as effector cells in both host defense and pathogenesis of allergic disease through the direct activation of TLR 4 and TLR2 on mast cells by microorganisms.

Mast cells represent a potential source of multifunctional cytokines that may participate in the recruitment and activation of other cells in the inflammatory microenvironment (27). Mast cells responded to PGN or LPS via TLR2 or TLR4, respectively. Mast cell responses to LPS are completely impaired in the absence of the TLR4 but not the TLR2 molecule, suggesting that TLR2 is not involved in LPS response in mast cells and that the LPS-induced TLR4 responsiveness was not affected by the existence of the TLR2 molecule. A reciprocal result was obtained in PGN-TLR2 stimulation of mast cells.

Interestingly, upon PGN stimulation, mast cells released more IL- 4 and IL- 5 , less TNF- $\alpha$, and no IL- $1 \beta$, even though TNF- $\alpha$ and IL-1 $\beta$ were predominant cytokines produced by mast cells upon LPS stimulation via TLR4 (18). In addition, PGN could induce degranulation of mast cells via TLR2 accompanied by $\mathrm{Ca}^{2+}$ influx, whereas the activation of mast cells by LPS via TLR4 did not lead to degranulation (18). So far, we still do not know how TLR2 is different from TLR4 in the signaling pathways of mast cell activation. To the extent that we analyzed the phosphorylation pattern of IкB- $\alpha$ and mitogen-activated protein kinases, we did not observe clear differences in TLR2 and TLR4 signaling pathways in mast cells (Figure 3 and data not shown), even though the severely impaired proinflammatory a

\section{Figure 4}

Mast cell TLR4 but not mast cell TLR2 is required for the full expression of innate immunity in a mast cell-dependent sepsis model. (a) $W / W^{2}$ mice without reconstitution or 5 weeks after reconstitution with BMMCs from $\mathrm{TLR}^{+/+}$, TLR4 $4^{-/}$, $\mathrm{TLR}^{+/+}$, or $\mathrm{TLR}^{-/-}$mice (6-16 mice per group) underwent CLP. The mortality was observed and expressed as percent survival. $W / W^{N}$ mice without $\mathrm{BMMC}$ reconstitution (injected with saline) were the control group. (b) Six hours after CLP, the mice were killed and peritoneal fluid was investigated. The total numbers of leukocytes and neutrophils were estimated by differential count and expressed as total cells per mouse. (c) The levels of indicated cytokines were measured by ELISA kit. Data shown are mean \pm SD of three mice.
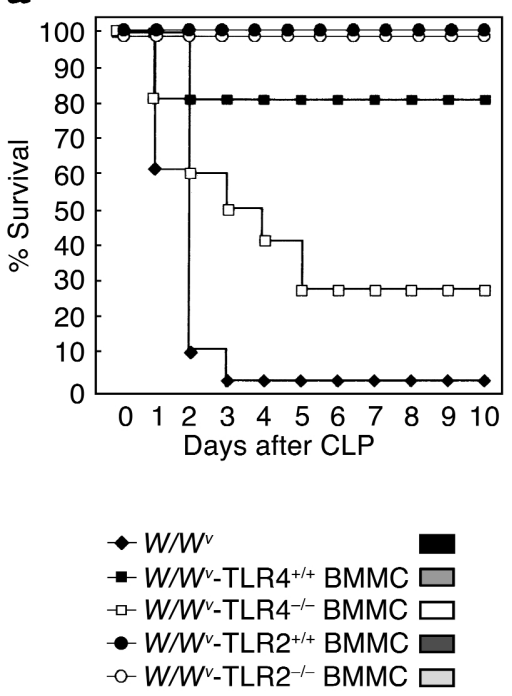
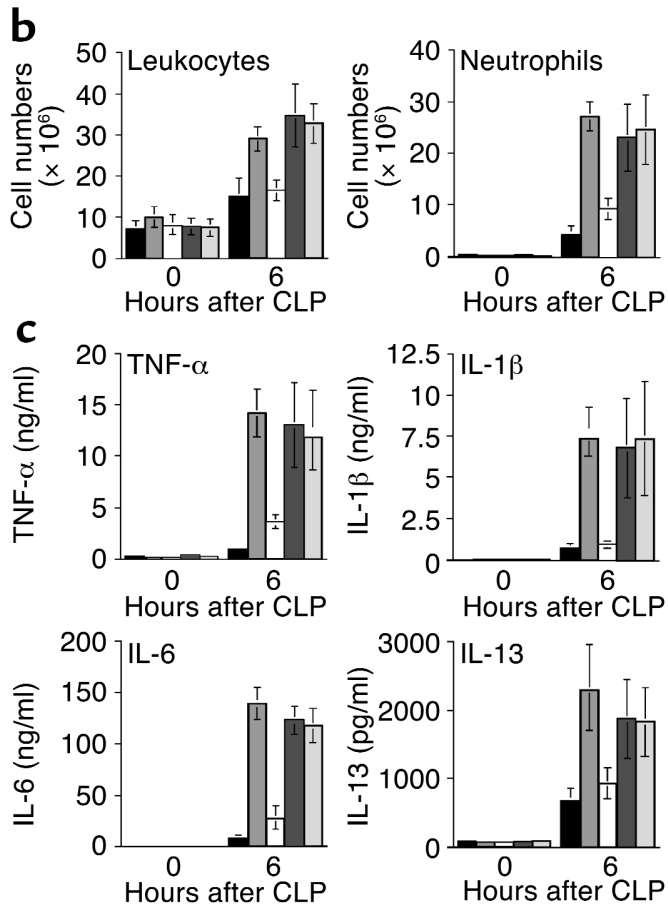
cytokine production of TLR2- or TLR4-deficient mast cells was correlated with the loss of NF- $\mathrm{KB}$ activation. Although the intracellular domain of TLRs is highly conserved in each TLR (29), recent findings suggest that downstream events are not always mediated by common components such as MyD88, an adaptor protein that links to the IL-1R-associated protein kinase and to the TNF receptor-activated factor $6(30,31)$. As TLR4 can activate both MyD88-dependent and -independent pathways that lead to different induction of genes in macrophages upon LPS stimulation (32-34), it is possible that TLR2 and TLR4 use different pathways for acti- vation of mast cells. While TLR4 recognizes relatively few ligands, TLR2 recognizes a wide variety of infectious pathogens (34). Since it is reported that ligand specificity and signal-transducing ability of TLR 2 are determined by heterodimeric interactions with other TLRs, such as TLR6 or TLR1 $(35,36)$, it would be useful to determine whether heterodimeric interaction of TLR2 and TLR6 is also required in mast cells. In particular, degranulation is a quite specific biological reaction confined to mast cells; it would be interesting to determine the TLR2-mediated specific signaling pathway that leads to this biological response. A recent report a

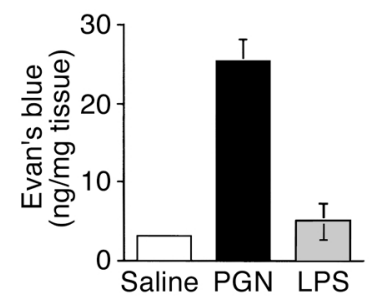

C

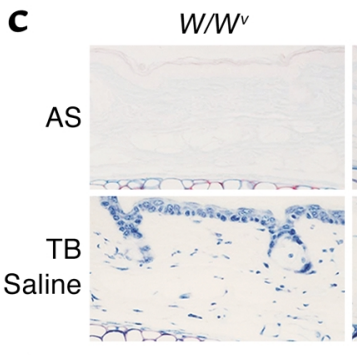

d

PGN

$W / W$
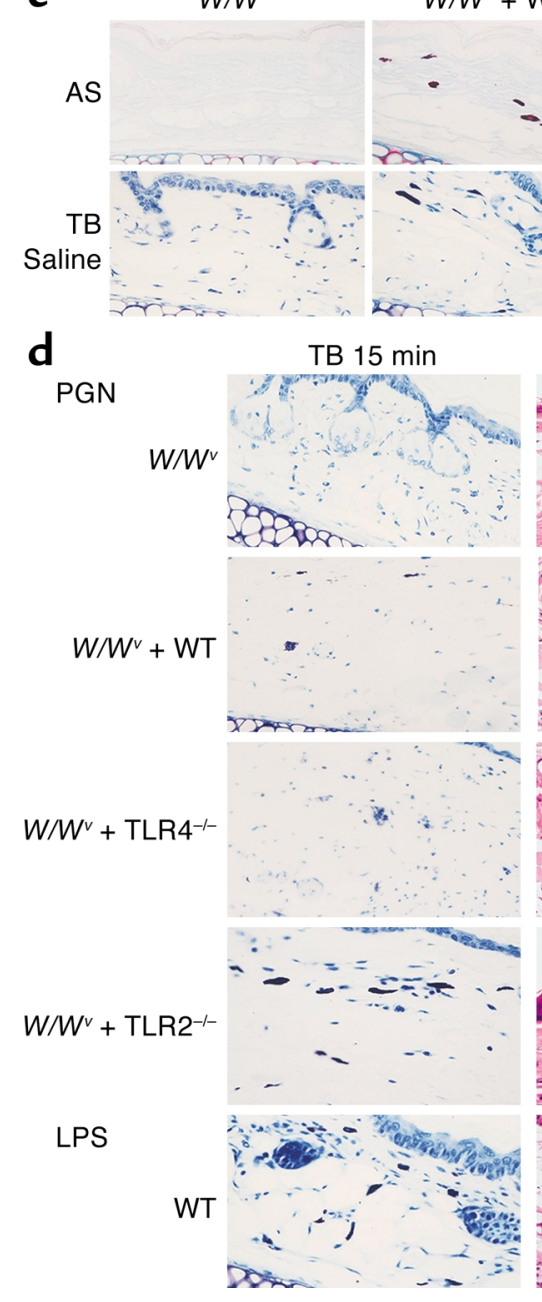

b

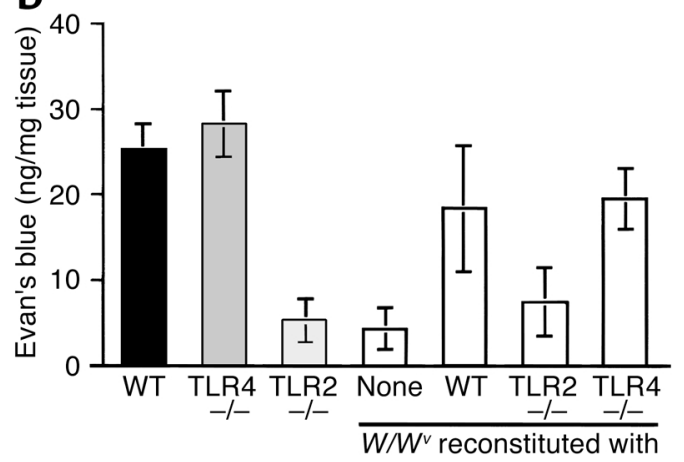

$W / W^{v}+W T$

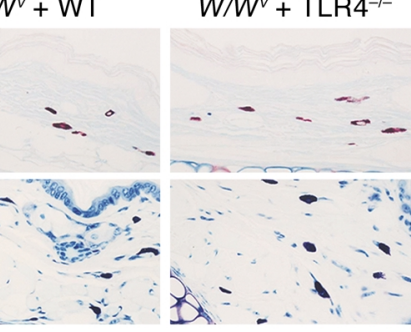

$W / W^{v}+T^{-1}$
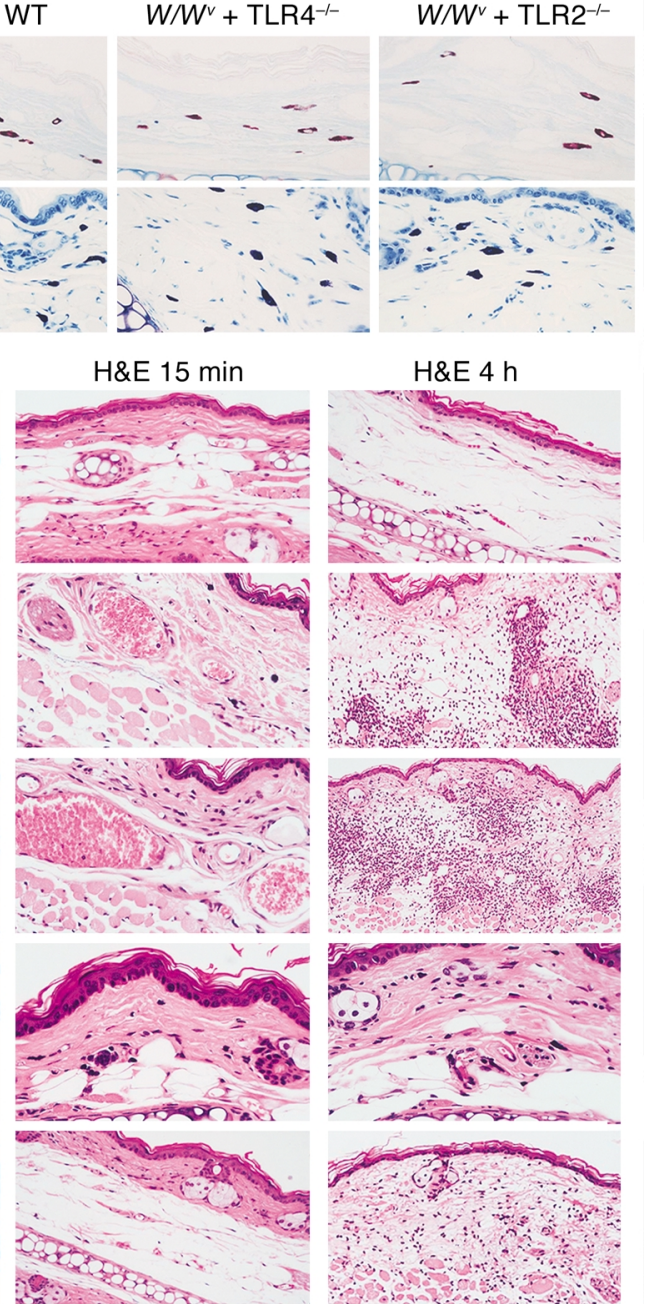

\section{Figure 5}

TLR2 on mast cell-dependent skin inflammation upon application of PGN. Reconstitution of skin mast cells of $W / W^{N}$ mice by $\mathrm{TLR}^{-/-}$, TLR4 ${ }^{-/-}$, or wild-type BMMCs was done as described in Methods. Wildtype mice (a) or indicated mice (b) were intravenously injected with $0.5 \%$ Evans blue 5 minutes before intradermal application and $20 \mu \mathrm{l}$ of PGN $(100 \mu \mathrm{g} / \mathrm{ml})$ or LPS $(100 \mathrm{ng} / \mathrm{ml})$ into the left ear or saline into the right ear. The mice were sacrificed 15 minutes after PGN or LPS injection, and dye contents in the tissues were measured as described in Methods. Data shown are mean \pm SD of three mice. (c) Reconstitution of skin mast cells of $W / W$ mice by TLR2-/-, TLR4 $4^{-/}$, or wild-type BMMCs was histologically confirmed by Alcian blue and Safranin staining (AS; without any treatment; upper panels) and Toluidine blue staining (TB; saline-injected; lower panels). (d) Mice were intradermally injected with PGN (upper four rows) or LPS (bottom row) as described above. Mice were sacrificed 15 minutes (left and middle columns) or 4 hours later (right column), and ear sections from $W / W$ (first row), $W / W^{2} /$ wild-type ( $W / W^{2} / \mathrm{WT}$; second row), $W / W^{N} /$ TLR $^{-/-}$(third row), or $W / W^{N} / T_{L R 2}{ }^{-/-}$mice (fourth row) were stained with $\mathrm{H} \& \mathrm{E}$ (middle and right columns) or Toluidine blue (TB; left column) for histological evaluations. All magnification is $\times 600$, except the figures at 4 hours after PGN application from $W / W^{N} / \mathrm{WT}$ and $W / W^{N} / \mathrm{TLR}^{-/-}$and at 4 hours after LPS application (all $\times 200)$. 
Table 1

TLR2-dependent activation of mast cells and infiltration of neutrophils in the skin upon PGN application

\begin{tabular}{|c|c|c|c|c|c|c|}
\hline \multicolumn{2}{|c|}{ Reagent applied } & \multicolumn{4}{|c|}{ PGN } & \multirow{2}{*}{$\begin{array}{l}\text { LPS } \\
\text { WT }\end{array}$} \\
\hline \multicolumn{2}{|c|}{ Mice } & $W / W^{2}$ & $W / W^{2}+W T$ & $W / W^{v}+\mathrm{TLR}^{-/-}$ & $W / W^{N}+\mathrm{TLR}^{-/-}$ & \\
\hline \multirow[t]{3}{*}{ Mast cell degranulation (\%) } & Extensive & 0 & $83.3 \pm 3.1$ & $86.3 \pm 4.7$ & $3.7 \pm 1.5$ & $2.3 \pm 0.6$ \\
\hline & Slight-Moderate & 0 & $11.0 \pm 4.6$ & $9.3 \pm 4.9$ & $16.3 \pm 3.1$ & $8.3 \pm 5.1$ \\
\hline & None & 0 & $5.7 \pm 4.0$ & $4.3 \pm 0.6$ & $80.0 \pm 3.6$ & $89.3 \pm 4.7$ \\
\hline \multirow[t]{2}{*}{ Neutrophils (no. $\left./ \mathrm{mm}^{2}\right)^{B}$} & $0 \mathrm{~h}$ & $1.3 \pm 1.2$ & $1.3 \pm 0.6$ & $1.7 \pm 0.6$ & $2.3 \pm 1.5$ & $2.7 \pm 1.2$ \\
\hline & $4 \mathrm{~h}$ & $3.7 \pm 0.6$ & $219.3 \pm 73.9 c$ & $198.7 \pm 57.3^{C}$ & $6.3 \pm 1.5$ & $36.3 \pm 8.5^{\mathrm{D}}$ \\
\hline
\end{tabular}

AMast cells were classified into three categories: extensively degranulated ( $50 \%$ of the cytoplasmic granules exhibiting fusion, staining alterations, and extrusion from the cell), slightly to moderately degranulated (10-15\% of the granules exhibiting fusion or discharge), or normal. ${ }^{\text {B }}$ The number of dermal neutrophils present at the reaction sites was determined as described in Methods and expressed as the number of cells per square millimeter of dermis. Data shown are mean $\pm \mathrm{SD}$ of three mice. ${ }^{C} P<0.01$ compared with the number of neutrophils in PGN-applied skin of $W / W$ and $W / W / T L R 2^{-1-}$. ${ }^{D} P<0.01$ compared with the number of neutrophils in PGN-applied skin of $W / W / W T$, and $W / W / T L R 4^{-/-}$.

suggests that JAK3 is a key regulator of mast cell-mediated innate immunity, especially TNF- $\alpha$ production from mast cells after $E$. coli stimulation and clearance of GNB (37). Thus, it would also be helpful to know the relationship of JAK3 activation and TLR signaling in mast cells upon stimulation with GNB.

Mast cell-deficient $\mathrm{WBB} \mathrm{F}_{1}-W / W^{v}$ mice and the model of "mast cell knock-in" mice could be used to analyze the roles of mast cells in biological response in vivo. Consistent with previous work (18), $\mathrm{WBB} \mathrm{F}_{1}-\mathrm{W} / \mathrm{W}^{v}$ mice were successfully reconstituted with BMMCs from genetically unmatched TLR $4^{+/+}$and TLR $4^{-/-}$, or TLR2 ${ }^{+/+}$ and TLR2 ${ }^{--}$mice that are a mixture of C57BL/ 6 and $129 \mathrm{SvJ}$ strains. During the study period, the reconstituted mice did not show any abnormality. The mast cells in both peritoneal cavity and skin 5 weeks after reconstitution were no different in number, ability to develop, and ability to degranulate in vitro. Therefore, the differences in the mortality of mice after CLP and in the acute skin reaction after PGN injection were not due to the difference in development of BMMCs in the $W / W^{\nu}$ environments. To determine why mast cells can survive and differentiate in genetically unmatched environments, further investigations are required.

We have previously demonstrated that BMMCs from $\mathrm{C} 3 \mathrm{H} / \mathrm{HeJ}$ mice, which had constitutive mutation of the intracellular domain of TLR4, show impaired response to LPS by means of cytokine production and NF- $\kappa B$ activation. Our present study using TLR $4^{-/-}$mice, which lack whole molecules of TLR4, had results similar to those of $\mathrm{C} 3 \mathrm{H} / \mathrm{HeJ}$ mice. This again clearly indicates that TLR4 but not TLR2 is the critical receptor for activation of mast cells by LPS both in vitro and in vivo. Compared with in vitro results where BMMCs from TLR4 ${ }^{-/}$mice completely lacked the LPS responses, determined by proinflammatory cytokine production and NF- $\kappa \mathrm{B}$ activation, it seemed that $W / W^{v} /$ TLR $4^{-/-}$mice showed a better survival rate than unreconstituted $W / W^{v}$ mice in the CLP model. These differences may reflect the involvement of other TLRs or other molecules, such as CD48 and C3 receptors, in activation of mast cells upon wholemicroorganism stimulation in vivo $(38,39)$.
Since PGN caused degranulation and cytokine production of mast cells in vitro, the role of mast cells in PGN-induced cutaneous acute response was investigated. Wheal reaction was observed within 5 minutes after PGN challenge, but not after vehicle injection, and was gradually enhanced after 15 minutes. We used a mast cell knock-in system with $W / W^{v}$ mice to exclude the possibility that mediator released from other endogenous cells that express TLR2 can cause increased vascular permeability. The skin reactions were observed within 5 minutes and were not observed in TLR2 ${ }^{-/-}$mice, $W / W^{v}$ mice, or $W / W^{v}$ mice reconstituted with TLR2-/- BMMCs, strongly suggesting that degranulation of mast cells through TLR2 activation was responsible for these reactions. In fact, we could observe the highly degranulated mast cells 15 minutes after PGN application in the skin of wild-type, $W / W^{v} / W T$, and $W / W^{v} /$ TLR4-/- mice, but not in the skin of TLR2 $2^{-/-}$and $W / W^{v} /$ TLR2 $2^{-/-}$mice, in the histological examination (Table 1). Furthermore, TLR4-/- mice and $W / W^{v} /$ TLR $4^{-/-}$mice showed responses similar to those of wild-type mice, suggesting that TLR4 (on mast cells or other cells) in the skin is not involved in PGN-induced vasodilatation. In addition, application of LPS in the skin did not lead to degranulation of mast cells or vasodilatation similar to that induced by PGN, suggesting that response to LPS in the skin has a biologically different character from that of PGN-induced response. Although it has been reported that mast cells can be activated by GPB through IgE bound on cells (40), this is, to our knowledge, the first evidence that shows the direct activation of mast cells via a specific pathogen recognition receptor, TLR2.

In summary, this study clearly shows that TLR4 but not TLR2 is the important molecule for mast cells in response to LPS challenge and that it functions in innate immunity against enterobacterial infection. In contrast, TLR2 but not TLR4 is responsible for activation of mast cells by $S$. aureus that might be involved in the pathogenesis of atopic dermatitis. Finding the microbial ligands and relevant receptors on mast cells, as well as signaling pathways that can activate mast cells, will elucidate the complexity of innate immunity and the roles of mast cells in conditions other than allergy. 
Also, the clarification of the mechanisms of mast cell activation by microorganisms through these TLRs may offer new insight into the treatment of allergic disease or inflammation in which bacterial infections and mast cell activations are common.

\section{Acknowledgments}

This work was supported in part by grants from the Ministry of Education, Science and Culture of the Japanese Government. V. Supajatura was supported by grants from Japan International Cooperation Agency. The authors thank K. Hoshino, H. Yagita, and K. Maeda for helpful discussions, and Y. Kanamaru and T. Uchida for technical assistance.

1. Metcalfe, D.D., Baram, D., and Mekori, Y.A. 1997. Mast cells. Physiol. Rev. 77:1033-1079.

2. Weber, S., et al. 1995. Mast cells. Int. J. Dermatol. 34:1-10.

3. Bradding, P., and Holgate, S.T. 1999. Immunopathology and human mast cell cytokines. Crit. Rev. Oncol. Hematol. 31:119-133.

4. Galli, S.J., and Wershil, B.K. 1996. The two faces of the mast cells. Nature. 381:21-22.

5. Malaviya, R., Ikeda, T., Ross, E., and Abraham, S.N. 1996. Mast cell modulation of neutrophil influx and bacterial clearance at sites of infection through TNF-alpha. Nature. 381:77-80.

6. Henz, B.M., Maurer, M., Lippert, U., Worm, M., and Babina, M. 2001. Mast cells as initiators of immunity and host defense. Exp. Dermatol. 10:1-10.

7. Kopp, E.B., and Medzhitov, Z. 1999. The Toll-receptor family and control of innate immunity. Curr. Opin. Immunol. 11:13-18.

8. Lien, E., et al. 2000. Toll-like receptor 4 imparts ligand-specific recognition of bacterial lipopolysaccharide. J. Clin. Invest. 105:497-504.

9. Hoshino, K., et al. 1999. Toll-like receptor 4 (TLR4)-deficient mice are hyporesponsive to LPS: evidence for TLR4 as the Lps gene product. J. Immunol. 162:3749-3752.

10. Poltorak, A., et al. 1998. Defective LPS signaling in $\mathrm{C} 3 \mathrm{H} / \mathrm{HeJ}$ and C57BL/10ScCr mice: mutations in Tlr4 gene. Science. 282:2085-2088.

11. Lien, E., et al. 1999. Toll-like receptor 2 functions as a pattern recognition receptor for diverse bacterial products. J. Biol. Chem. 274:33419-33425.

12. Takeuchi, O., et al. 1999. Differential roles of TLR2 and TLR4 in recognition of Gram-negative bacterial cell wall components. Immunity. 11:443-451.

13. Brightbill, H.D., et al. 1999. Host defense mechanisms triggered by microbial lipoproteins through toll-like receptors. Science. 285:732-736.

14. Aliprantis, A.O., et al. 1999. Cell activation and apoptosis by bacterial lipoproteins through Toll-like receptor-2. Science. 285:736-739.

15. Schwandner, R., Dziarski, R., Wesche, H., Rothe, M., and Kirschning, C.J. 1999. Peptidoglycan- and lipoteichoic acid-induced cell activation is mediated by Toll-like receptor 2. J. Biol. Chem. 274:17406-17409.

16. Yoshimura, A., et al. 1999. Cutting edge: recognition of Gram-positive bacterial cell wall components by the innate immune system occurs via Toll-like receptor 2. J. Immunol. 163:1-5.

17. Means, T.K., et al. 1999. Human Toll-like receptors mediate cellular activation by Mycobacterium tuberculosis. J. Immunol. 163:3920-3927.

18. Supajatura, V., et al. 2001. Protective roles of mast cells against enterobacterial infection are mediated by Toll-like receptor 4. J. Immunol. 167:2250-2256.

19. Mihm, M.C.J., Soter, N.A., Dvorak, H.F., and Austen, K.F. 1976. The structure of normal skin and the morphology of atopic eczema. J. Invest. Dermatol. 67:305-312.

20. Damsgaard, T.E., Olesen, A.B., Sorensen, F.B., Thestrup-Pedersen, K., and Schiotz, P.O. 1997. Mast cells and atopic dermatitis. Stereological quantification of mast cells in atopic dermatitis and normal human skin. Arch. Dermatol. Res. 289:256-260.

21. Nakahata, T., Spicer, S.S., Cantey, J.R., and Ogawa, M. 1992. Clonal assay of mouse mast cell colonies in methylcellulose culture. Blood. 60:352-361.

22. Nakano, T., et al. 1985. Fate of bone marrow-derived cultured mast cells after intracutaneous, intraperitoneal, and intravenous transfer into genetically mast cell-deficient $W / W^{v}$ mice: evidence that cultured mast cells can give rise to both connective tissue type and mucosal mast cells. J. Exp. Med. 162:1025-1043.

23. Tsai, M., et al. 2000. In vivo immunological function of mast cells derived from embryonic stem cells: an approach for the rapid analysis of even embryonic lethal mutaions in adult mice in vivo. Proc. Natl. Acad. Sci. USA. 97:9186-9190.

24. Inagaki, N., Goto, S., Nagai, H., and Koda, A. 1985. Pharmacological characterization of mouse ear PCA. Int. Arch. Allergy Appl. Immunol. 78:113-117.

25. Yano, H., Wershil, B.K., Arizono, N., and Galli, S.J. 1989. Substance P-induced augmentation of cutaneous vascular permeability and granulocyte infiltration in mice is mast cell dependent. J. Clin. Invest. 84:1276-1286.

26. Wershil, B.K., Wang, Z.S., Gordon, J.R., and Galli, S.J. 1991. Recruitment of neutrophils during IgE-dependent cutaneous late phase reactions in the mouse is mast cell-dependent. Partial inhibition of the reaction with antiserum against tumor necrosis factor $\alpha$. J. Clin. Invest. 87:446-453.

27. Gordon, J.R., Burd, P.R., and Galli, S.J. 1990. Mast cells as a source of multifunctional cytokines. Immunol. Today. 11:458-464.

28. Anderson, K.V. 2000. Toll signaling pathways in the innate immune response. Curr. Opin. Immunol. 12:13-19.

29. Gay, N.J., and Keith, F.J. 1991. Drosophila Toll and IL-1 receptor. Nature. 351:355-356

30. Medzhitov, R, et al. 1998. MyD88 is an adaptor protein in the hToll/IL-1 receptor family signaling pathways. Mol. Cell. 2:253-258.

31. Weshe, H., Henzel, W.J., Shillinglaw, W., Li, S., and Cao, Z. 1997. MyD88: an adaptor that recruits IRAK to the IL-1 receptor complex. Immunity. 7:837-847.

32. Kawai, T., Adachi, O., Ogawa, T., Ikeda, K., and Akira, S. 1999. Unresponsiveness of MyD88-deficient mice to endotoxin. Immunity. 11:115-122.

33. Kaisyo, T., and Akira, S. 2001. Dendritic-cell function in Toll-like receptor-and MyD88-knockout mice. Trends Immunol. 22:78-83.

34. Akira, S., Takeda, K., and Kaisho, T. 2001. Toll-like receptors: critical proteins linking innate and acquired immunity. Nat. Immunol. 2:675-680.

35. Ozinsky, A., et al. 2000. The repertoire for pattern recognition of pathogens by the innate immune system is defined by cooperation between toll-like receptors. Proc. Natl. Acad. Sci. USA. 97:13766-13771.

36. Takeuchi, O., et al. 2001. Discrimination of bacterial lipoproteins by Toll-like receptor 6. Int. Immunol. 3:933-940.

37. Malaviya, R., Navara, C., and Uckun, F.M. 2001. Role of Janus kinase 3 in mast cell-mediated innate immunity against gram-negative bacteria. Immunity. 18:313-321.

38. Malaviya, R., Gao, Z., Thankavel, K., Van der Merwe, P.A., and Abraham, S.N. 1999. The mast cell tumor necrosis factor alpha response to FimHexpressing Escherichia coli is mediated by the glycosylphosphatidylinositol-anchored molecule CD48. Proc. Natl. Acad. Sci. USA. 96:8110-8115.

39. Prodeus, A.P., Zhou, X., Maurer, M., Galli, S.J., and Carroll, M.C. 1997. Impaired mast cell-dependent natural immunity in complement C3-deficient mice. Nature. 390:172-175.

40. Genovese, A., et al. 2000. Bacterial immunoglobulin superantigen protein $\mathrm{A}$ and $\mathrm{L}$ activate human heart mast cells by interacting with immunoglobulin E. Infect. Immun. 68:5517-5524. 